\title{
Combination of engineering, technical and design solutions on the example of the renewable energy sources placement on the building envelope
}

\author{
Natalia Galkina*, Maksim Govorunov, Genrikh Muro, and Sergey Khalezin \\ Don State Technical University, 344010, Rostov-on-Don, Russia
}

\begin{abstract}
Renewable energy design is a complex process requiring many different factors to consider. There are many methods for calculating solar heat supply systems, varying in the degree of feasibility of each specific solution relative to its counterparts. Moreover, each design stage is based solely on the determination of the engineering and technical parameters of the selected solar system execution scheme; such important indicators as reliability, load-bearing capacity and operational safety of structural elements on which the elements of solar power plants are located often fade into the background and are even rarely taken into account by agreement with specialists performing related sections (architectural treatment - AT; structural concept - SC). In turn, the latter creates the need to revise the already adopted design options, and sometimes recalculate the developed measures. The solution to this problem is the development of a conceptual design methodology, including the calculation of active solar systems, taking into account not only climatic parameters that affect the performance of solar systems, but also the fundamental influencing factors that form the load on the load-bearing structural elements of the building, as well as entail a change in it. energy model (in particular, thermal protection indicators). At the same time, it is also possible to correlate the architectural and artistic appearance of the building with the accepted layout of the heliofields of active solar systems on the facades and other protruding elements of the building; including inclined facades.
\end{abstract}

\section{Introduction}

Solar energy is one of the leading renewable energy sources (RES) [1]. This is explained by the presence of such functions as energy inexhaustibility, high environmental friendliness, availability and comfort of operation of solar systems, innovation, low cost of heat or electric energy received, as well as a relatively insignificant degree of development in this direction, which is characterized by the possibility of further, almost unhindered increase in efficiency indicators and active solar systems quality $[2,3]$.

\footnotetext{
${ }^{*}$ Corresponding author: natash-galkina@mail.ru
} 
The emergence of solar energy falls on the first half of the 20th century and is characterized by rapid growth. In Russia, this direction originates with some delay from the world start, nevertheless, it can be proud of significant successes in the area under consideration, which is the merit, first of all, of a wide circle of Soviet scientists and specialists who turned their eyes and interests to solar energy - who considered its potential and the opportunities it bestowed. Unfortunately, since that time, domestic science in the field of solar energy has undergone a significant decline, which can rightfully be described as extinction. However, this does not mean that solar systems are not used in Russia, their equipment and elements do not have localized production in northern Asia and eastern Europe, and the introduction of solar power plants has low feasibility in these regions. Undoubtedly, the southern regions of Russia are the warmest and sunniest, suitable for the use of solar heat and power supply systems. Moreover, their efficiency factor for specific cases, sometimes it is in no way inferior to analogs located in the most favorable latitudes (equatorial countries).

First of all, the development of special, often individual, characterized by an unconventional and non-trivial approach to the calculation, installation and operation of a solar plant contributes to the achievement of high efficiency and quality, as well as the feasibility of introducing a specific design option for an active solar system [4]. Limited in opportunities, Russian specialists and scientists actively apply their knowledge and skills in the field of improving the existing elements of solar systems, as well as the development and implementation of new, previously unused methods for the device and execution of circuit solutions of installations, and, moreover, a combination of previously adopted methods and solutions, which often border to a greater extent not on science, but with gumption. While the Western contingent of specialists relies on personal experience and achievements, focusing on attempts to introduce fundamentally new developments (inventions), Russian RI borrows the experience of foreign colleagues, transforming and modernizing existing innovative solutions, correlated with domestic results.

First of all, it is worthwhile to understand that there are two main innovative approaches to achieving a higher result, characterized by an increase in indicators - an increase in efficiency, quality and feasibility [5]. The first is the improvement of previously adopted and developed equipment and individual elements of engineering systems, as well as methods of calculation, design and implementation of the results. The second is the development of fundamentally new, previously unused and unparalleled innovative solutions, methods, as well as devices and systems in general. Moreover, each of these options has its own positive and negative features, distinguishable and amenable to characterization directly when assessing each specific case separately.

The design of active solar systems, like any other engineering and technical or construction complex of measures, is accompanied by the inherent - obligatory need to take into account various influencing factors, which in their main variety, in the existing generally accepted calculation methods, are reduced to taking into account the climatic indicators and the resulting operating parameters of the systems [6]. At the same time, such important aspects as the placement of equipment, devices and elements of the adopted wiring diagram of the solar plant are reduced to a conditional, often unfounded and not taking into account the structural characteristics of the building, location. In turn, this may be a consequence of a number of problems, accompanied by the likelihood of a decrease in building safety indicators and the formation of a high threat to the life and health of citizens during operation. Nevertheless, taking into account these indicators the adopted conceptual methodology for designing solar energy supply systems is reduced to a simple strengthening of already existing structures, by selecting materials and elements with a greater bearing capacity, which, undoubtedly, is characterized by the achievement of the set 
result that meets the current standards and rules [7], but has a noticeably lower feasibility and quality level in comparison with other possible variations.

Thus, we can conclude that the active solar systems elements introduction requires correlation and complex consideration of the structural conditions for placement, as well as the possibility of not cascading - sequential development of design solutions (both engineering and technical, and architectural and planning), but parallel, providing for the totality and interdependence of the selected design solutions, that is, deviating from the generally accepted methodology for performing the design stage of construction, the joint production of sections for the development of design and engineering measures. This approach gives an opportunity to achieve higher quality indicators, energy efficiency and the feasibility of the chosen design option, including due to the possibility of taking into account the changing indicators of the thermal protection of the building (in particular, increasing the resistance to heat transfer).

The achievement of the above-mentioned result is proposed to be carried out, as already mentioned earlier, by joint (parallel) development at the design stage of architectural and planning and engineering solutions. An excellent illustrative example of such work is the variation in the placement of active solar systems radiation-receiving elements (solar collectors and panels) on building structures (facades, roofs), engineering and technical measures focused on the absolute correlation of both sections, in order to achieve the highest quality and energy efficiency [8]. At the same time, a separate, key aspect of the subsequent study, the authors of the article propose to consider the possibility of placing solar collectors and solar panels on inclined facades of buildings, previously appropriately prepared for such an implementation.

\section{Discussion}

The installation of beam receiving devices for solar systems should be envisaged from the conditions for ensuring such operating parameters of solar installations in which the needs of subscribers are fully satisfied during the billing periods (warm or year-round). At the same time, there are a number of basic measures, the variation of which allows achieving the required indicators. These include:

1) increase in the receiving surface of solar cells (see Fig. 1);

2) replacement of the adopted solar cells with more efficient analogs;

3) orientation of the building facades on which solar cells are placed (collectors or panels);

4) slope of facades (including roofs) on which solar cells are placed;

5) the orientation of the solar cells themselves;

6) solar cell tilt.

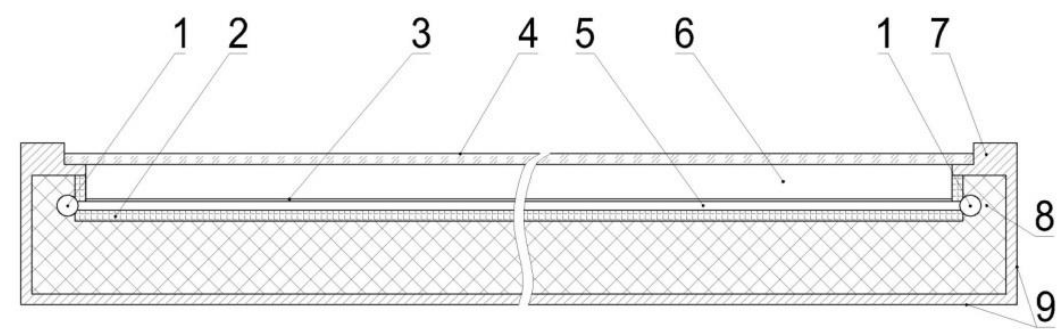

Fig. 1. Flat solar collector design:

1 - manifold distribution pipe; 2 - reflective coating under the absorbent panel; 3 - absorbent panel (radiation receiving surface); 4 - translucent coating (glass); 5 - absorbent panel tube; 6 - air or airless 
(vacuum) space; 7 - compactors; 8 - thermal insulation; 9 - case.

The first two measures are costly and always lead to an increase in the cost of the solar system, and, at the same time, to a decrease in the coefficient of technical and economic feasibility. However, one way or another, accounting and application of all of the abovementioned measures can significantly increase the efficiency of any thermal and electric solar plant. Nevertheless, the options 3-6 in some cases have less cost [9] and no less efficiency. At the same time, the existing configuration of the building, in the conditions of urban development, does not always have an area of facades sufficient for the installation of a large area solar cells. It is required to take into account and vary other indicators - the correct orientation and location of solar cells, which, of course, are basic and trivial aspects in the design of active solar systems, but not always fully realizable. The main operation principle in this case is the correct orientation of solar cells in the vertical and horizontal axes, which directly affects the amount of solar radiation entering the receiving surface $[10]$.

The latter, in turn, is achieved by varying the location of the building at the design stage - the device of one of its facades (including the roof) maximally oriented to the correct angle for a particular latitude [11]. Also, in the case of placing beam receiving devices on inclined elements of facades - roofs, the inclination angle of their slopes should be varied, if possible. The result of such manipulations, as mentioned earlier, will be a significant increase in the efficiency of the solar plant with a constant area of solar cells (collectors or panels).

However, these measures are not always properly implemented, especially in the existing buildings' conditions. Nearby buildings, including, if they are taller, can overlap the facade required for the device [12]. Also, when heliofields are introduced on flat roofs, difficulties arise with the placement of radiation receiving devices associated with the equipment location of other building's engineering systems - ventilation, air conditioning, etc. In other words, there are various factors, one way or another, hindering the achievement of the established result.

To be able to solve these problems, the authors of this article propose the introduction of inclined facades at the design stage as one of the most likely options for application, which will result in an increase in the area potentially suitable for placing solar cells even if the angle of horizontal orientation is insufficient. In other words, a symbiosis of architectural planning and engineering solutions is proposed when designing buildings with active solar systems (see Fig. 2).

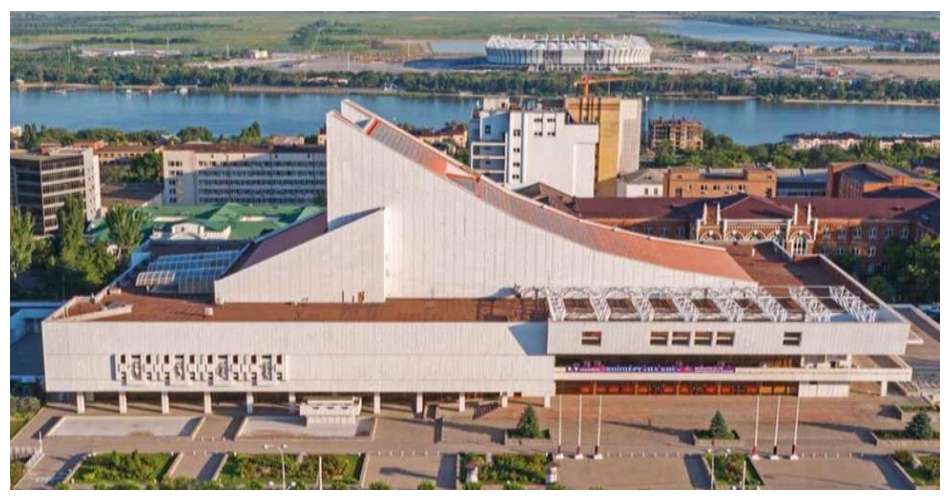

Fig. 2. An example of a possible variation in a building facade slope 


\section{Results}

When configuring architectural, planning and engineering solutions in the above-shown way, location of the beam receiving devices on adjacent facades from the optimal one with a slight decrease in the final efficiency is possible. In other words, if the existing building does not allow one of the building facades to be accurately oriented to the optimal angle, then it is possible to arrange an inclined facade with the subsequent arrangement of beam receiving devices on it with a different angle of the vertical orientation inclination. Thus, it is possible to increase the operating parameters of solar plants. At the same time, replacing a flat roof or part of it with an inclined one significantly increases the area suitable for placing solar cells.

Among other things, the above-mentioned configuration has the advantage that entails not only an increase in work efficiency, but also an increase in the thermal protection of the building $[13,14]$. When located on a flat roof or on strictly vertical facades, solar cells are installed on special supporting structures that act as bases, including those forming the correct inclination angle of the vertical orientation (see Fig. 3). In the case of a facade with a deliberately required angle of inclination, there is no need to use such structures - solar cells (collectors and panels) are immediately built into the covering or placed on it (see Fig. 4), tightly adhering to the latter.

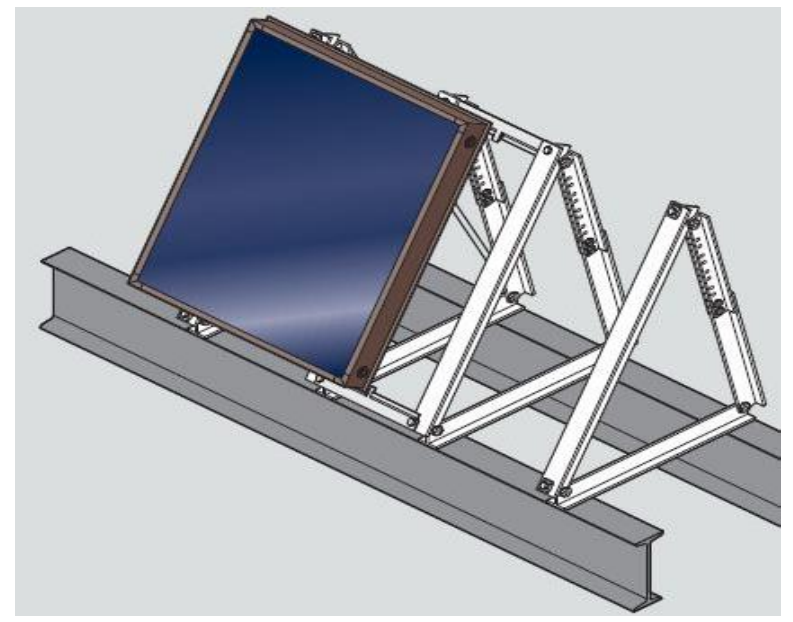

Fig. 3. Support structure for the solar collector location

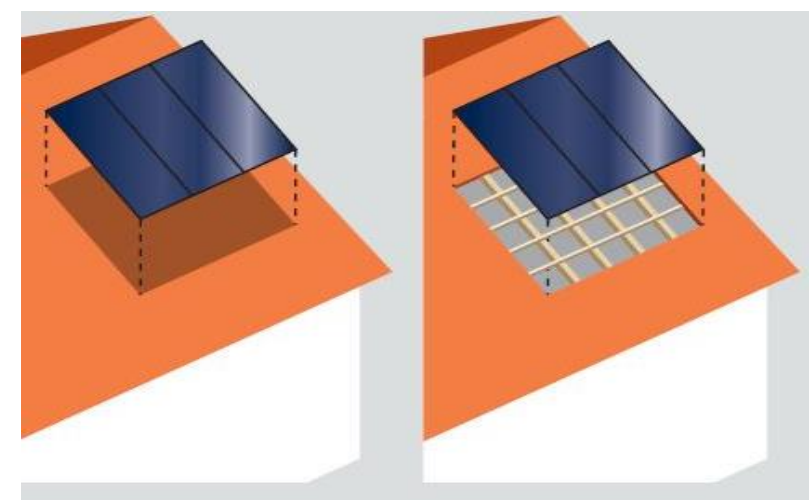

Fig. 4. Installation principle of solar collectors built into the roof covering 
In this case, the plane on which the solar collectors or panels are located obviously has the required inclination angle, due to which the area suitable for placement and the system efficiency actually increase (Fig. 5).

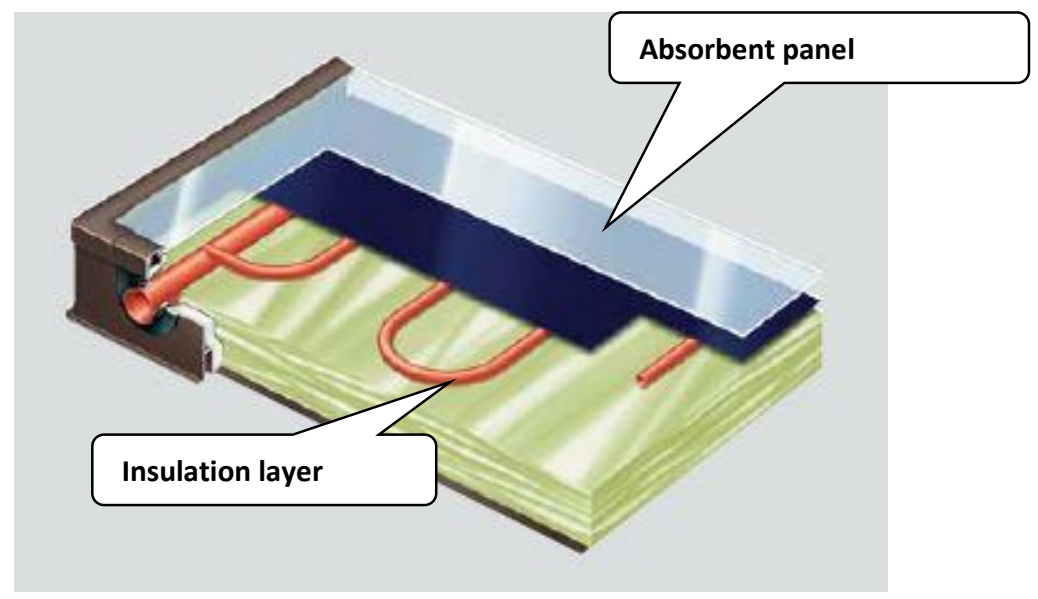

Fig. 5. The location of the insulation under the absorbing panel of the solar collector

In this case, the plane on which the solar collectors or panels are located obviously has the required inclination angle, due to which the area suitable for placement and the system efficiency actually increase (Fig. 6).

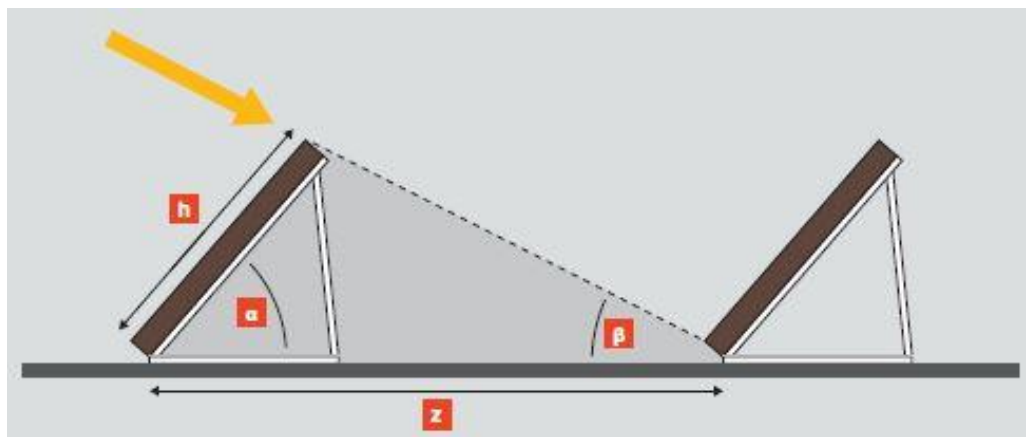

Fig. 6. Arrangement of solar collectors in the horizontal plane:

$\mathrm{z}$ - manifold row spacing; $\mathrm{h}$ - collector height; $a$ - collector tilt angle; $\beta$ - the solstice angle

In the case described above, the increase in the thermal protection of the building is realized by introducing the elements that actively absorb solar radiation into the external building envelopes (BE), which increases the BE surface temperature, due to which the building thermal energy losses are locally reduced. At the same time, the operating indicators of the solar heat supply system remain practically unchanged $[15,16]$. At the same time, solar collectors have a layer of insulation located under the absorbent panel (Fig. 5). This structural element of the device additionally increases the thermal insulation degree of the external enclosing structure directly at the solar collector location.

When implementing all the above-stated aspects, the structural characteristics of new structures should be taken into consideration as well as the loads generated by the weight of the devices located, which can have a significant mass, depending on the configuration and installation methods, especially in the case of solar heating systems, which provide for the 
presence of a coolant. For this, it is necessary to carry out the calculations for the strength and stability of structures provided for by the current rules and regulations [17].

At the same time, it is also worth remembering that the process of introducing the inclined facades is accompanied by a change in the wind and snow impact parameters on the supporting structures of the building. In this case, it is necessary to objectively evaluate the reliability and performance parameters of certain elements in certain conditions, to distribute and transfer loads. Such an important indicator as the safety of a construction facility cannot be neglected.

First of all, each specific case should be analyzed for the possibility of introducing other possible design and technical solutions that ensure maximum technical and economic feasibility while maintaining safe operation and absent risks, including the object destruction and the formation of a threat to human life and health [18].

One of the possible options in this case is the additional load-bearing structures introduction, such as columns, which take the load from the inclined facades and transfer them to the lower horizontal and vertical load-bearing structures. Including, in this case, the development of design and technical documentation is also integral, and subsequently, acceptance work aimed at quality control and acceptance of critical structures [19].

\section{Conclusion}

Implementation of the above-noted concept allows achieving the highest efficiency in the active solar systems' design. The result of such work, taking into account the aspects mentioned earlier, is the provision of such technical and economic indicators as reliability, energy efficiency, economic benefit, Technosphere safety and innovative solutions.

Introduction of active solar systems forms a reduction in the traditional fuel sources consumption, which reduces the amount of harmful emissions into the atmosphere, and provided that these systems are correctly designed and constructed, it is possible, among other things, to achieve high performance indicators, with a constant amount of capital investment [20].

\section{References}

1. N. Aldibekov, Renewable energy sources and energy saving (nuclear power plant, Almaty, 2016)

2. J.A. Duffy and U. Beckman, The basics of solar thermal energy (Intellect, Dolgoprudny, 2013)

3. Ya. Markhotsky, Basics of ecology and energy saving (Vyshaya Shk., Minsk, 2014)

4. N. Stoyanov, Complex energy supply of isolated facilities from solar energy, (North Caucasian Federal Units, Stavropol, 2014)

5. A. Pirozhnikova, M. Govorunov, T. Pirozhnikova and G. Muro, IOP Conference Series: Materials Science and Engineering (2019)

6. B. Germanovich and A. Turilin, Alternative energy sources and energy saving (Science and Technology, St. Petersburg, 2014)

7. V. Adamovich, B. Barhin, Va. Varezhkin and other, Architectural design of public buildings and structures (Strojizdat, 2014)

8. A. Dzindzela and A. Sizjakin, Efficient use of low-potential heat energosberezhenie 54-57 (2012)

9. N. Vil'chik, The architecture of the buildings (Gostekhizdat, Moscow, 2016) 
10. The NASA Surface Meteorology and Solar Energy. 2019. https://power.larc.nasa.gov/data-access-viewer/.

11. O. Samarin, Thermophysics, energy saving and energy efficiency (DIA, Moscow, 2014)

12. N. Gerasimov, AVOK 28-33 (2014)

13. V. Belyaev, Yu. Granik and Yu. Matrosov, Energy efficiency and heat protection of buildings (DIA, Moscow: 2012)

14. SP50.13330.2012. Thermal protection of buildings. Updated edition of SNiP 23-022003, Moscow: Ministry of Regional Development of Russia, 2012, 98.

15. O. Samarin, Issues of Economics in the Microclimate of Buildings (ASV Moscow, 2015)

16. N. Galkina, O. Gurova and M. Govorunov, The use of BEM technologies for the design of active solar heat supply systems that ensure technosphere and environmental safety, unpublished.

17. SP 70.13330.2012. Bearing and enclosing structures. The updated edition of SNiP 3.03.01-87 (as amended No.1, 3) (Moscow6 Gosstroy, FAU "FTSS", 2013).

18. Federal Law "Technical Regulations on the Safety of Buildings and Structures," (State Duma of the Russian Federation, Moscow 2009)

18. SP 48.13330.2019 "Organization of construction. Actualized edition of SNiP 12-012004". (with Changes \#1), Moscow, Ministry of Construction and Housing and Communal Services of the Russian Federation, 2019.

19. V. Bespalov, O. Gurova, E. Lysova and others, Development and Choice of an Evidence-based Technique of the Most Ecologically Effective and Energetically Active Economic Technologies of Cleaning of Toxic Components of the Departing and Exhaust Gases of Objects of an Urban Environmental Assessment, BIOSCIENCES BIOTECHNOLOGY RESEARCH ASIA 1459-1470 (2015) 